\title{
A PDA-based System development and implementation for Manufacturing Execution System
}

\author{
Xin Wang \\ Mechanical engineering and automation institute \\ Beijing University of Aeronautics and Astronautics \\ Beijing, China \\ e-mail: wangxin_buaa@hotmail.com
}

\begin{abstract}
This paper describes a PDA-based mobile system of workpiece data inquiring and acquisition that can process data by calling the Web Service. The system is practically tested and verified in a MES project, the result shows that it can provide the effective product information through scanning bar code, and improve the rapid reaction capability and work efficiency greatly.
\end{abstract}

\section{Keywords-PDA; Web Service; MES; Windows CE}

\section{INTRODUCTION}

MES (Manufacturing Execution System) is aimed to strengthen the execution capacity of MRP (Material Requirement Planning), and connects MRP with workshop field control. Fist, MES have higher requirements to realtime and completeness for the data. Meanwhile the environment of fieldwork collection is complex, especially for certain discrete location. Then, Use what means to realize the acquisition of field data to the server to drive the normal running of MES other modules becomes the key problem [1].

PDA (Personal Digital Assistant) is a kind of mobile handheld digital equipment which can provide information management services for users [2]. It has the characteristics of simple stability, high reliability, compact and lightweight, easy to carry and low price, especially the combination of PDA and mobile communication technology brings more broad application prospects[3][4].

This paper designs and implements a data inquiring and acquisition system based on PDA that can process data with production management database by calling the Web Service. According to the purpose of project, complete the division of function modules of the system, hardware and software design and system configuration. At last, the system is practically used in a MES project and can meet the needs of workshop field.

\section{PURPOSE}

This system is designed for the operation of workshop workers, production monitor or the leadership of department and realizing the following functions:

- Obtaining products and components information through scanning bar code.

- $\quad$ Structuring information channel from Web Service to database and PDA client.

\author{
Guangrong Yan \\ Mechanical engineering and automation institute \\ Beijing University of Aeronautics and Astronautics \\ Beijing, China \\ e-mail: yangr@buaa.edu.cn
}

- Convenience operation and easy maintaining with function extensions.

\section{SYSTEM DESIGN}

The system includes three sections: PDA client-side, Web Service server-side, database server. PDA client-side scans bar code to get process card number and query database server through remote calling of Web Service. Then return work piece information to PDA interface. Meanwhile users can save information to database in a similar way. The data relation schema is shown in Fig 1.

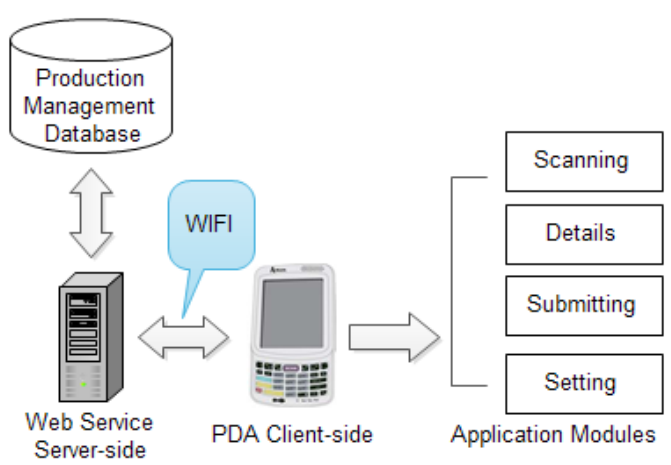

Figure 1. The schema of system structure

\section{DEVELOPMENT ENVIROENT}

A. Hardware

ARGOX PT 90(Windows Embedded CE 6.0, WIFI), Web Server, Database Server

B. Software

Microsoft Visual Studio 2008, Microsoft SQL Server 2008, PT90_CE600 SDK, Device Emulate

\section{Programming Language}

C\#, .NET 3.5

\section{INPLEMENTATION}

According to purpose the project includes two sections: Web Server-side and PDA Client-side. In PDA Client, there are mainly four function modules respectively named 
"Scanning Module”, "Details Module”, “Submitting Module" and "Setting Module".

\section{A. Web Server-side}

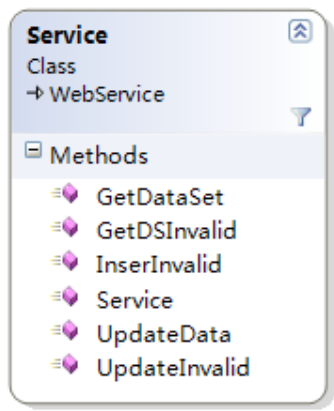

Figure 2. Web Service Methods

Web Server-side is a middle layer in the system to connect and communicate between Client-side and Database-side. In Web Server-side a series of function methods are defined such as querying or modifying work piece information, inserting a new record, etc. as shown in Fig 2.

\section{B. Scanning Module}

The bar code is custom made according to the actual requirement and can be scanned to character string by PDA device. A bar code example is shown in fig 3 .

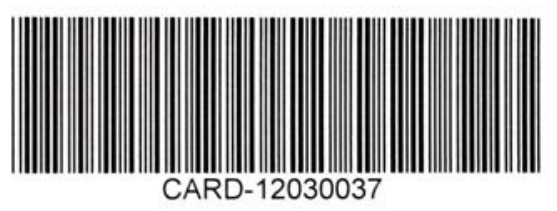

Figure 3. A bar code used in workpiece

Scanning Module (Fig 4) interface is loaded when PDA client starts. The cursor is positioned in textbox to wait scanning. While users click on the "SCAN" key in PDA keyboard aiming a bar code, the process card number will display in the textbox and device automatically enter into querying status with calling Web Service method to search database through wireless communication. If users manually input process card number, clicking the "ENTER" key in PDA keyboard or "Reading" button in the interface can also achieve the same effect.

\section{Details Module}

Details Module's (Fig 4) function is to display the query result of Scanning Module interface. The information shown in the interface contains "Task ID", "Work Piece Name", "Process Card Number" in textboxes and the working procedure list in a data grid view. When users click a record in data grid or double click "Submit" button on screen display, system will jump to Submitting Module interface.
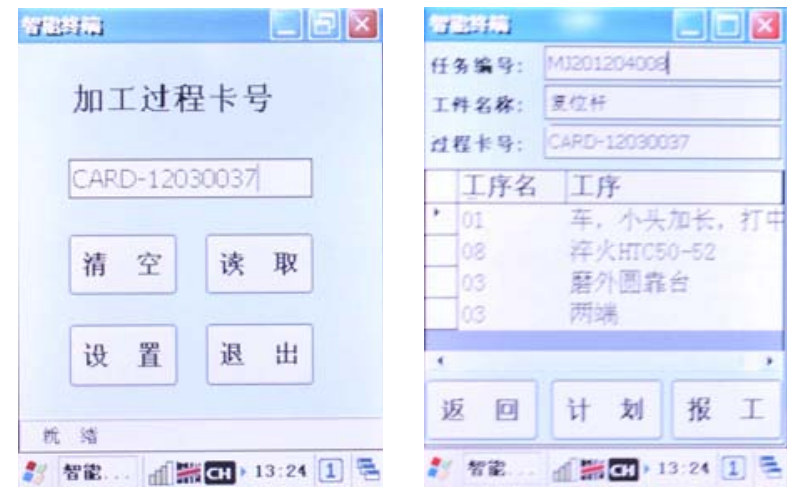

Figure 4. Scanning Module and Details Module interfaces

D. Submitting Module

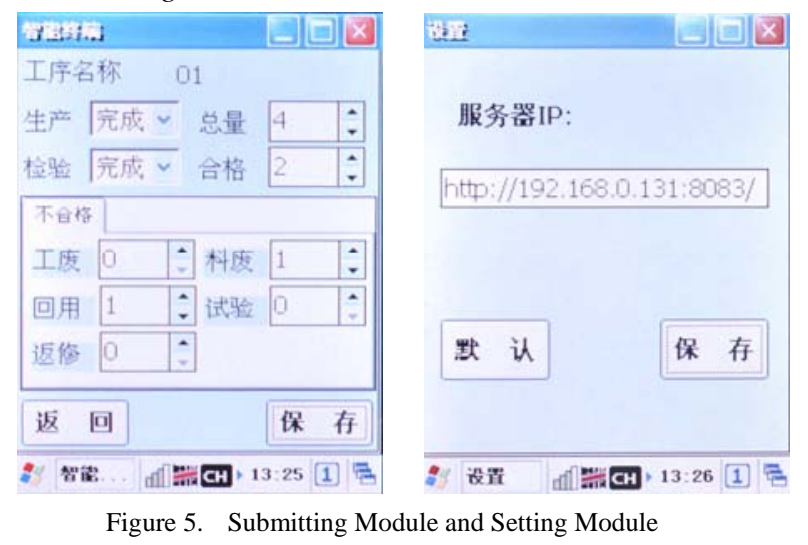

This module's (Fig 5) function is to submit user's modification of the MES database. The display mode includes "status of production", "status of inspect", "amount of production", "amount of inspect" and disqualification status. All of the contents are queried and shown in advance. Users can alter and save values by clicking buttons on screen according to certain rules and constraints which must be conformed or else popping error message.

\section{E. Setting Module}

Setting Module (Fig 5) is used for setting Web Service IP address for user according to Actual requirement. When inputting a new address and clicking the save button, the IP address can be configured in system.

\section{VITAL TECHNOLOGY}

\section{A. PDA's Dynamic Call to Web Server}

In the practical application, the Web Service IP address used by PDA may transform Along with working environment changes. So we must realize the Dynamic call to Web Server.

This system get the IP address in Setting Module and save to a global variable named "strServer". When Web Service is required, the following codes are executed for passing variable:

Service sv = new Service (strServer); 
Then the service of sv can be called and implemented in system. This method must have two preconditions: 1 . Web server selected needs to have been published the same Web Service with default server; 2 . in the "reference.cs" folder the construction function named "Service (string url)" should be altered as "this.Url = url;"

\section{B. Data Grid's Register in WinCE System}

In Details Module interface, the data grid's content is filled from the dataset queried from database directly. But there are only two fields requiring display in grid. To solve this problem, the system adopted the method as altering column width value to " -1 " in order to hide the column. Then the data of grid can be called invisible. One example is shown as follows:

//define grid style

DataGridTableStyle ts = new DataGridTableStyle () ; ts.MappingName $=$ dtMain. TableName;

//define column style

DataGridColumnStyle gcs $=$ new DataGrid TextBox Column (); gcs.MappingName = "PROCESSID"; gcs.HeaderText = "PROCESSID"; gcs. Width = -1; ts.GridColumnStyles.Add (gcs);

//fill data grid this.dataGrid1.TableStyles.Add (ts); this.dataGrid1.DataSource = dtMain;

\section{Multiple Controls' Operation in WinCE System}

In Submitting Module interface, it refers to save multiple controls' values with certain rules and constraints. The flowchart of program is shown as Fig 6 .

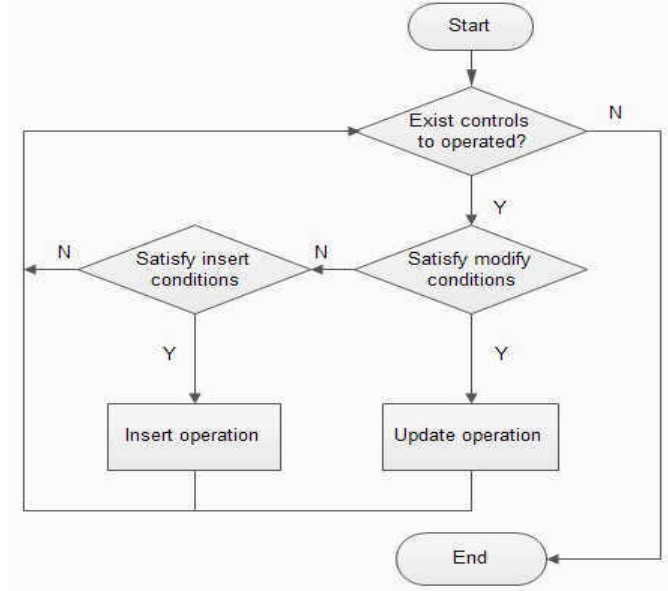

Figure 6. The flowchart of multiple controls' operation

This system defines four arrays respectively to store different flags, and then executed inserting or modifying operation according to these flags. The critical codes are shown as follows:

//the flag of database contained

List $<$ int $>$ KeyList $=$ new List $<$ int $>()$; //the flag of controls modified

List $<$ int $>$ ChangeList $=$ new List $<$ int $>()$;

//the controls to be operated

NumericUpDown [] Nus = new NumericUpDown [] \{...\}; //the names controls related

string [] keyName $=$ new string []$\{\ldots\}$;

//execute

for (int listNO = 1 ; listNO $<6$; listNO++)

\{

decimal amount $=$ Nus [listNO -1$].$ Value;

$/ /$ modify data

if (KeyList.Contains (listNO) \&\& ChangeList.

Contains (listNO)) $\{\ldots\}$

//insert data

else if (KeyList.LastIndexOf (listNO) $<0$ \&\&

ChangeList.Contains (listNO) \&\& amount $!=0)\{\ldots\}$

\}

In addition, "KeyList" is inserted when obtaining data, "ChangeList" is inserted when "NumericUpDown_ ValueChanged” events responding. Thus system can judge inserting or modifying with the monitoring the two statuses. The array representation of controls can achieve cycle operation of multiple controls and optimized the codes.

\section{CONCLUSION}

A PDA-based data requiring and acquisition system is designed in the paper according to MES needs. It can collect workpiece data and transmit it into database. In the actual use, the system has a good user experience performances and makes workpiece information obtaining more convenience and quickly. As the users' practice with the system, improvement approaches are necessary. More interaction functions and modules are needed to extend, so that it can further satisfy the demand of the users in manufacturing industry.

\section{REFERENCES}

[1] CHANG Da-liang, ZHANG Xiao-Yu, PENG Hui, and SHI Hai-bo, The Appliance of Web Service in MES Data Transmission, Microcomputer Information, 2011,27(1):139-141.

[2] J Zhang, X Chen, J Yang et al, “A PDA-based Sign Translator”, Proceedings of IEEE International Conference on Multimodal Interfaces. 2002: 217-222.

[3] Pai-Tsun Cheng, Li-Min Tsai, Li-Wei Lu, et al, "The Design of PDA-based Biomedical Data Processing and Analysis for Intelligent Wearable Health Monitoring Systems,"Proc. IEEE Symp. International Conference on Computer and Information Technology (CIT 04), IEEE Press, Sept. 2004, pp. 879-884, doi: 10. 1109/CIT. 2004.1357306.

[4] Y-W Chen, Z-J Yan, J-C Huang, et a1, "Implementation of aPDA/GPS Based Development Platform and Its Application in Native Education”, Proceedings of the IEEE International Conference on Communications, Circuits and Systems and West Sino Expositions. 2002, 2:1556-1560.

[5] V Gopailakrishna, N Kehtarnavaz and P Loizou, "Real-Time PDABased Recursive Fourier Transform Implementation for Cochlear Implant Applications," Proc. IEEE Symp. International Conference on Acoustics, Speech and Signal Processing(ICASSP 09), IEEE Press, Apr. 2009, pp. 1333-1336, doi:10.1109/ICASSP. 2009.4959838. 